PROCEEDINGS OF THE

AMERICAN MATHEMATICAL SOCIETY

Volume 140, Number 8, August 2012, Pages 2589-2597

S 0002-9939(2011)11169-9

Article electronically published on December 1, 2011

\title{
TRANSFER MAPS AND PROJECTION FORMULAS
}

\author{
GONÇALO TABUADA
}

(Communicated by Brooke Shipley)

\begin{abstract}
Transfer maps and projection formulas are undoubtedly one of the key tools in the development and computation of (co)homology theories. In this paper we develop a unified treatment of transfer maps and projection formulas in the non-commutative setting of dg categories. As an application, we obtain transfer maps and projection formulas in algebraic $K$-theory, cyclic homology, topological cyclic homology, and other scheme invariants.
\end{abstract}

\section{TRANSFER MAPS}

A differential graded $(=d g)$ category, over a base commutative ring $k$, is a category enriched over complexes of $k$-modules (morphism sets are complexes) in such a way that composition fulfills the Leibniz rule $: d(f \circ g)=(d f) \circ g+(-1)^{\operatorname{deg}(f)} f \circ(d g)$; see Appendix $\mathrm{A}$ for details. Dg categories solve many of the technical problems inherent to triangulated categories and are nowadays widely used in algebraic geometry, in representation theory of quiver algebras, and even in mathematical physics; see Keller's ICM address [9].

Recall from [9, §4.6] that a $\operatorname{dg}$ functor $F: \mathcal{A} \rightarrow \mathcal{B}$ is a called a derived Morita equivalence if the restriction of scalars functor $\mathcal{D}(\mathcal{B}) \stackrel{\sim}{\rightarrow} \mathcal{D}(\mathcal{A})$, between the associated derived categories, is an equivalence of (triangulated) categories. As proved in [19, Thm. 5.3] the category dgcat of small dg categories carries a Quillen model structure whose weak equivalences are precisely the derived Morita equivalences. The homotopy category hence obtained will be denoted by Hmo. Thanks to Toën [24, Cor. 4.8] (and [19, Rk. 5.11]) the Hom-sets of Hmo can be described as $\operatorname{Hom}_{\mathrm{Hmo}}(\mathcal{A}, \mathcal{B}) \simeq \operatorname{Iso} \operatorname{rep}(\mathcal{A}, \mathcal{B})$, where Iso denotes the set of isomorphism classes, and $\operatorname{rep}(\mathcal{A}, \mathcal{B})$ the full subcategory of $\mathcal{D}\left(\mathcal{A}^{\mathrm{op}} \otimes \mathbb{L} \mathcal{B}\right)$ consisting of those $\mathcal{A}$ - $\mathcal{B}$-bimodules $X$ such that for every object $a \in \mathcal{A}$ the $\mathcal{B}$-module $X(-, a)$ is a compact object 14 in the derived category $\mathcal{D}(\mathcal{B}) ;-\otimes^{\mathbb{L}}-$ stands for the derived tensor product of $\mathrm{dg}$ categories. The localization functor dgcat $\rightarrow \mathrm{Hmo}$ is the identity on objects and sends a $\operatorname{dg}$ functor $F: \mathcal{A} \rightarrow \mathcal{B}$ to the isomorphism class of the $\mathcal{A}$ - $\mathcal{B}$-bimodule $(a, b) \mapsto \mathcal{B}(b, F(a))$.

The above description of the Hom-sets suggests that any functor defined on dg categories which inverts derived Morita equivalences should be endowed with an extra degree of "contravariant functoriality" with respect to the following class of dg functors.

Received by the editors June 25, 2010 and, in revised form, March 4, 2011.

2000 Mathematics Subject Classification. Primary 18D20, 19D55, 14F05.

Key words and phrases. Transfer maps, projection formulas, dg categories, algebraic $K$-theory, cyclic homology, topological cyclic homology, scheme invariants.

The author was partially supported by the FCT-Portugal grant PTDC/MAT/098317/2008. 
Definition 1.1. Let $\mathcal{T} r$ be the class of dg functors $F: \mathcal{A} \rightarrow \mathcal{B}$ such that for every object $b \in \mathcal{B}$ the $\mathcal{A}$-module $a \mapsto \mathcal{B}(F(a), b)$ is a compact object in the derived category $\mathcal{D}(\mathcal{A})$.

Example 1.2. Natural examples of elements in $\mathcal{T} r$ are given by morphisms $f: A \rightarrow$ $B$ of unital (but not necessary commutative) $k$-algebras such that $B$ is projective of finite type as an $A$-module; we consider $A$ and $B$ as dg categories with a single object. More generally, we can consider morphisms such that $B$ admits a finite resolution by projective $A$-modules of finite type. Other examples, of a schemetheoretical nature, are described in Lemma 3.1.

Theorem 1.3. (i) Let $E:$ dgcat $\rightarrow \mathrm{D}$ be a functor which inverts derived Morita equivalences. Then, for every $F \in \mathcal{T} r$ we have an associated transfer map $E^{t r}(F)$ : $E(\mathcal{B}) \rightarrow E(\mathcal{A})$. Moreover this procedure is functorial, i.e. $E^{\operatorname{tr}}\left(\mathrm{id}_{\mathcal{A}}\right)=\mathrm{id}_{E(\mathcal{A})}$ and $E^{t r}(G \circ F)=E^{t r}(F) \circ E^{\operatorname{tr}}(G)$.

(ii) Let $\eta: E_{1} \Rightarrow E_{2}$ be a natural transformation between derived Morita invariant functors. Then $\eta$ is compatible with the transfer maps; i.e. for every $F \in \mathcal{T} r$ we have the equality $\eta_{\mathcal{A}} \circ E_{1}^{\operatorname{tr}}(F)=E_{2}^{\operatorname{tr}}(F) \circ \eta_{\mathcal{B}}$.

Proof. The homotopy category $\mathrm{Hmo}$ is the localization of dgcat with respect to the class of derived Morita equivalences. Therefore, since by hypothesis the functor $E$ inverts derived Morita equivalences, we obtain a well-defined functor $\bar{E}$ making the following diagram commutative:

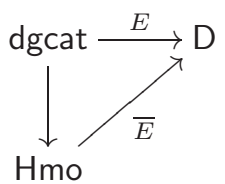

Now, let $F: \mathcal{A} \rightarrow \mathcal{B}$ be an arbitrary dg functor. Associated to $F$ we have not only the $\mathcal{A}$ - $\mathcal{B}$-bimodule

$$
X_{F}: \mathcal{A} \otimes^{\mathbb{L}} \mathcal{B}^{\mathrm{op}} \longrightarrow \mathcal{C}_{\mathrm{dg}}(k) \quad(a, b) \mapsto \mathcal{A}(b, F(a))
$$

but also the $\mathcal{B}$ - $\mathcal{A}$-bimodule

$$
{ }_{F} X: \mathcal{B} \otimes^{\mathbb{L}} \mathcal{A}^{\mathrm{op}} \longrightarrow \mathcal{C}_{\mathrm{dg}}(k) \quad(b, a) \mapsto \mathcal{B}(F(a), b) .
$$

Clearly, $X_{F}$ belongs to $\operatorname{rep}(\mathcal{A}, \mathcal{B})$. In contrast, ${ }_{F} X$ belongs to $\operatorname{rep}(\mathcal{B}, \mathcal{A})$ if and only if for every $b \in \mathcal{B}$ the $\mathcal{A}$-module $a \mapsto \mathcal{B}(F(a), b)$ is a compact object in the derived category $\mathcal{D}(\mathcal{A})$. Note that this condition is precisely the one of Definition 1.1. which implies that $F$ belongs to $\mathcal{T} r$ if and only if the bimodule ${ }_{F} X$ belongs to $\operatorname{rep}(\mathcal{B}, \mathcal{A})$. In particular, if $F$ belongs to $\mathcal{T} r$ we obtain a well-defined morphism $\left[{ }_{F} X\right]$ in $\operatorname{Hom}_{\mathrm{Hmo}}(\mathcal{B}, \mathcal{A})=\operatorname{Iso} \operatorname{rep}(\mathcal{B}, \mathcal{A})$. The transfer map $E^{\operatorname{tr}}(F)$, associated to $F$, is then the morphism in $\mathrm{D}$ given by $\bar{E}\left(\left[{ }_{F} X\right]\right)$. Given small dg categories $\mathcal{A}, \mathcal{B}$ and $\mathcal{C}$, the composition operation in $\mathrm{Hmo}$ corresponds to the derived tensor product of bimodules

$$
\text { Iso } \operatorname{rep}(\mathcal{A}, \mathcal{B}) \times \operatorname{Iso} \operatorname{rep}(\mathcal{B}, \mathcal{C}) \longrightarrow \operatorname{Iso} \operatorname{rep}(\mathcal{A}, \mathcal{C}) \quad([X],[Y]) \mapsto\left[X \otimes_{\mathcal{B}}^{\mathbb{L}} Y\right] .
$$

Hence, if $G: \mathcal{B} \rightarrow \mathcal{C}$ is another dg functor in $\mathcal{T} r$, we have a natural isomorphism ${ }_{(G \circ F)} X \simeq{ }_{F} X \otimes_{\mathcal{B}}^{\mathbb{L}}{ }_{G} X$ of $\mathcal{C}$ - $\mathcal{A}$-bimodules, which implies that ${ }_{(G \circ F)} X$ belongs to $\operatorname{rep}(\mathcal{C}, \mathcal{A})$. We obtain then the equality $\left[{ }_{(G \circ F)} X\right]=\left[{ }_{F} X\right] \circ\left[{ }_{G} X\right]$ in Hmo and consequently the equality $E^{\operatorname{tr}}(G \circ F)=E^{\operatorname{tr}}(F) \circ E^{\operatorname{tr}}(G)$ in D. This concludes the proof of item (i). 
Let us now prove item (ii). Since the functors $E_{1}$ and $E_{2}$ are derived Morita invariant, the natural transformation $\eta$ between $E_{1}$ and $E_{2}$ is also a natural transformation between the induced functors $\overline{E_{1}}$ and $\overline{E_{2}}$; see the above diagram (1.4). Hence, the equalities

$$
E_{1}^{t r}(F)=\overline{E_{1}}\left(\left[{ }_{F} X\right]\right), \quad E_{2}^{t r}(F)=\overline{E_{2}}\left(\left[{ }_{F} X\right]\right)
$$

allow us to conclude that $\eta$ is compatible with the transfer maps, i.e. for every $F \in \mathcal{T} r$ we have the equality $\eta_{\mathcal{A}} \circ E_{1}^{t r}(F)=E_{2}^{t r}(F) \circ \eta_{\mathcal{B}}$.

Connective algebraic $K$-theory $(K)$, non-connective algebraic $K$-theory $(\mathbb{K})$, Hochschild homology $(H H)$, cyclic homology $(H C)$, negative cyclic homology $(H N)$, periodic cyclic homology $(H P)$, topological Hochschild homology $(T H H)$, and topological cyclic homology $(T C)$ are all examples of derived Morita invariant functors; see 11, 10, 17, 21, 23. Therefore, by Theorem 1.3(i) we obtain transfer maps:

$$
\begin{array}{cl}
K_{*}^{t r}(F): K_{*}(\mathcal{B}) \rightarrow K_{*}(\mathcal{A}), & \mathbb{I}_{*}^{t r}(F): \mathbb{K}_{*}(\mathcal{B}) \rightarrow \mathbb{K}_{*}(\mathcal{A}), \\
H H_{*}^{t r}(F): H H_{*}(\mathcal{B}) \rightarrow H H_{*}(\mathcal{A}), & H C_{*}^{t r}(F): H C_{*}(\mathcal{B}) \rightarrow H C_{*}(\mathcal{A}), \\
H N_{*}^{t r}(F): H N_{*}(\mathcal{B}) \rightarrow H N_{*}(\mathcal{A}), & H P_{*}^{t r}(F): H P_{*}(\mathcal{B}) \rightarrow H P_{*}(\mathcal{A}), \\
T H H_{*}^{t r}(F): T H H_{*}(\mathcal{B}) \rightarrow T H H_{*}(\mathcal{A}), & T C_{*}^{t r}(F): T C_{*}(\mathcal{B}) \rightarrow T C_{*}(\mathcal{A}) .
\end{array}
$$

When $F$ is as in Example 1.2, the transfer map $K_{*}^{t r}(F)$ coincides with Quillen's original transfer map $f_{*}: K_{*}(B) \rightarrow K_{*}(A)$ on algebraic $K$-theory; see [15] page 103] and [25, $\S \mathrm{V}, 3.3 .2]$. Another important consequence of Theorem 1.3(ii) is the fact that the above transfer maps are compatible with the classical higher Chern characters and with the Dennis trace map

$$
c h_{*, i}: K_{*} \Rightarrow H C_{*+2 i}, \quad c h_{*}^{-}: K_{*} \Rightarrow H N_{*}, \quad D t r_{*}: K_{*} \Rightarrow T H H_{*} .
$$

For a description of these natural transformations consult [12, §8.4, §11.4].

Remark 1.11. Although the unified treatment of transfer maps given in this paper was not available in the literature, transfer maps and their compatibility with natural transformations have been "around" for a long time; see for instance the work of Bökstedt-Hsiang-Madsen [2], Hesselholt-Madsen [8], Keller [1], Schlichtkrull [16], and others.

\section{Projection FORMUlas}

The tensor product $-\otimes-$ of $k$-algebras extends naturally to dg categories, giving rise to a symmetric monoidal structure on dgcat. In this section we assume that $D$ is a symmetric monoidal triangulated category (with unit object $\mathbf{1}$ ), $E$ : dgcat $\rightarrow \mathrm{D}$ is a symmetric monoidal derived Morita invariant functor, and $F: \mathcal{A} \rightarrow \mathcal{B}$ is an element in $\mathcal{T} r$ between dg categories endowed with a "multiplication"

$$
m_{\mathcal{A}}: \mathcal{A} \otimes \mathcal{A} \longrightarrow \mathcal{A}, \quad m_{\mathcal{B}}: \mathcal{B} \otimes \mathcal{B} \longrightarrow \mathcal{B} .
$$

Given an object $P$ in $\mathrm{D}$, we will denote by $\operatorname{Hom}_{*}(\mathbf{1}, P)$ the $\mathbb{Z}$-graded abelian group of morphisms such that $\operatorname{Hom}_{n}(\mathbf{1}, P):=\operatorname{Hom}\left(\Sigma^{n}(\mathbf{1}), P\right)$.

Theorem $1.3(i)$ furnishes us a transfer map $E^{\operatorname{tr}}(F)$, and so by composition with $E(F)$ and $E^{\operatorname{tr}}(F)$ we obtain homomorphisms

$$
\begin{aligned}
E(F)_{*}: \operatorname{Hom}_{*}(\mathbf{1}, E(\mathcal{A})) & \longrightarrow \operatorname{Hom}_{*}(\mathbf{1}, E(\mathcal{B})), \\
E^{t r}(F)_{*}: \operatorname{Hom}_{*}(\mathbf{1}, E(\mathcal{B})) & \longrightarrow \operatorname{Hom}_{*}(\mathbf{1}, E(\mathcal{A}))
\end{aligned}
$$


of $\mathbb{Z}$-graded abelian groups. Note that since $E$ is symmetric monoidal, the "multiplication" dg functors (2.1) endow the $\mathbb{Z}$-graded abelian groups $\operatorname{Hom}_{*}(\mathbf{1}, E(\mathcal{A})$ ) and $\operatorname{Hom}_{*}(\mathbf{1}, E(\mathcal{B}))$ with a bilinear multiplicative operation -. - . If the "multiplications" (2.11) are moreover associative, commutative, or admit a unit object, the same holds for the multiplicative operations on $\operatorname{Hom}_{*}(\mathbf{1}, E(\mathcal{A}))$ and $\operatorname{Hom}_{*}(\mathbf{1}, E(\mathcal{B}))$. However, the statement and proof of our general projection formula does not make use of these extra properties.

Thanks to Theorem 1.3 $(i)$, the functor dgcat $\rightarrow$ Hmo is endowed with transfer maps. Let us denote by $\left[X_{F}\right]$ the image of $F$ in $\mathrm{Hmo}$ and by $\left[{ }_{F} X\right]$ the associated transfer map. Under the above assumptions we have the following result.

Proposition 2.4. Let $F$ be an element in $\mathcal{T} r$ making the following diagrams commutative:

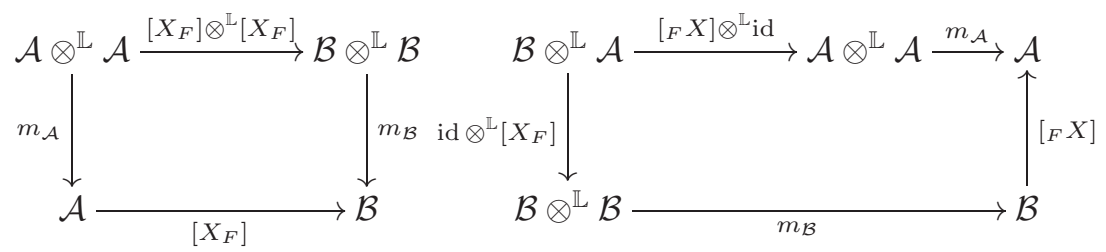

Then, for every $x \in \operatorname{Hom}_{*}(\mathbf{1}, E(\mathcal{B}))$ and $y \in \operatorname{Hom}_{*}(\mathbf{1}, E(\mathcal{A}))$ the following projection formula

$$
E^{\operatorname{tr}}(F) \circ(x \cdot(E(F) \circ y))=\left(E^{\operatorname{tr}}(F) \circ x\right) \cdot y
$$

holds in $\operatorname{Hom}_{*}(\mathbf{1}, E(\mathcal{A}))$.

Proof. Since the functor $E$ sends derived Morita equivalences to isomorphisms, we obtain a well-defined functor $\bar{E}$ as in diagram (1.4). Moreover, since by hypothesis $E$ is symmetric monoidal, so is $\bar{E}$. Therefore, when we apply the functor $\bar{E}$ to the right-hand side diagram in (2.5) and use the equalities

$$
E(F)=\bar{E}\left(\left[X_{F}\right]\right), \quad E^{t r}(F)=\bar{E}\left(\left[{ }_{F} X\right]\right),
$$

we obtain the following commutative diagram in $\mathrm{D}$ :

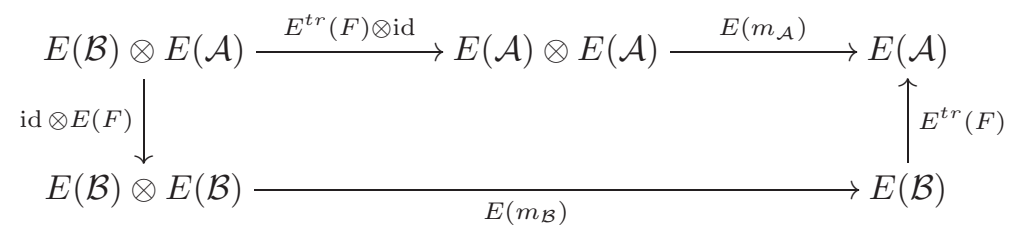

Now, let $x \in \operatorname{Hom}_{n}(\mathbf{1}, E(\mathcal{B}))$ and $y \in \operatorname{Hom}_{m}(\mathbf{1}, E(\mathcal{A}))$. Using the symmetric monoidal structure on D we obtain an element $x \otimes y$ in $\operatorname{Hom}_{n+m}(\mathbf{1}, E(\mathcal{B}) \otimes E(\mathcal{A}))$. Thanks to the above diagram (2.7) we have the following equality:

$$
E^{t r}(F) \circ E\left(m_{\mathcal{B}}\right) \circ(\mathrm{id} \otimes E(F)) \circ(x \otimes y)=E\left(m_{\mathcal{A}}\right) \circ\left(E^{t r}(F) \otimes \mathrm{id}\right) \circ(x \otimes y) .
$$

Recall that the multiplicative operations -.- on $\operatorname{Hom}_{*}(\mathbf{1}, E(\mathcal{B}))$ and $\operatorname{Hom}_{*}(\mathbf{1}, E(\mathcal{A}))$ are obtained by combining the monoidal structure on D with the maps $E\left(m_{\mathcal{A}}\right)$ and $E\left(m_{\mathcal{B}}\right)$. Therefore, we observe that the above equality (2.8) coincides with the projection formula

$$
E^{t r}(F) \circ(x \cdot(E(F) \circ y))=\left(E^{\operatorname{tr}}(F) \circ x\right) \cdot y .
$$

The proof is then finished. 
Note that the commutativity of the left-hand side diagram in (2.5) implies that the homomorphism (2.2) respects the multiplicative operations. Hence, the $\mathbb{Z}$ abelian group $\operatorname{Hom}_{*}(\mathbf{1}, E(\mathcal{B}))$ carries a $\operatorname{Hom}_{*}(\mathbf{1}, E(\mathcal{A}))$-action and so the projection formula (2.6) entails that the homomorphism (2.3) is $\operatorname{Hom}_{*}(\mathbf{1}, E(\mathcal{A}))$-linear.

Example 2.9. Natural examples of elements in $\mathcal{T} r$ which satisfy the conditions of Proposition 2.4 are given by the morphisms $f: A \rightarrow B$ presented in Example 1.2 between commutative $k$-algebras. In this commutative case the multiplication operations in $A$ and $B$ are $k$-algebra homomorphisms, and so we obtain dg functors as in (2.1). The commutativity of the left-hand side diagram in (2.5) is clear. The commutativity of the right-hand side diagram in (2.5) follows from the natural isomorphism $B \otimes_{B} A \otimes_{A} B \simeq B \otimes_{A} A$ of $A$-modules. Scheme-theoretical examples are described in Proposition 3.4

We now give some examples of functors which can be expressed as $\operatorname{Hom}_{*}(\mathbf{1}, E(-))$ for certain symmetric monoidal functors $E$.

Example 2.10 (Algebraic $K$-theory). Recall from [18, §10, §15] the construction of the universal additive and localizing invariant of dg categories

$$
\mathcal{U}_{\mathrm{dg}}^{\text {add }}: \operatorname{dgcat} \longrightarrow \operatorname{Mot}_{\mathrm{dg}}^{\text {add }}(e), \quad \mathcal{U}_{\mathrm{dg}}^{\text {loc }}: \operatorname{dgcat} \longrightarrow \operatorname{Mot}_{\mathrm{dg}}^{\text {loc }}(e) .
$$

Here, $\operatorname{Mot}_{\mathrm{dg}}^{\text {add }}(e)$ and $\operatorname{Mot}_{\mathrm{dg}}^{\text {loc }}(e)$ are the categories of non-commutative motives in the additive and localizing settings; see [4, 5, 18, for details. These triangulated categories carry symmetric monoidal structures, with unit objects $\mathcal{U}_{\mathrm{dg}}^{\text {add }}(\underline{k})$ and $\mathcal{U}_{\mathrm{dg}}^{\mathrm{loc}}(\underline{k})$, making the above universal invariants symmetric monoidal. Thanks to [18. Thm. 15.10] and [4. Thm. 7.16] we have, for every small dg category $\mathcal{C}$, natural isomorphisms of $\mathbb{Z}$-graded abelian groups

$$
\operatorname{Hom}_{*}\left(\mathcal{U}_{\mathrm{dg}}^{\text {add }}(\underline{k}), \mathcal{U}_{\mathrm{dg}}^{\text {add }}(\mathcal{C})\right) \simeq K_{*}(\mathcal{C}), \quad \operatorname{Hom}_{*}\left(\mathcal{U}_{\mathrm{dg}}^{\text {loc }}(\underline{k}), \mathcal{U}_{\mathrm{dg}}^{\text {loc }}(\mathcal{C})\right) \simeq K_{*}(\mathcal{C}) .
$$

If $\mathcal{C}$ is moreover endowed with a "multiplication" dg functor as in (2.1), then $K_{*}(\mathcal{C})$ and $\mathbb{K}_{*}(\mathcal{C})$ inherit a multiplicative operation. For instance, if $A$ is a commutative algebra as in Example 2.9, the multiplicative operation on $K_{*}(A)$ is the one described in [12, §11.2.16]; see [22, Ex. 2.5]. It follows then from Proposition 2.4 applied to $E=\mathcal{U}_{\mathrm{dg}}^{\text {add }}$ that $x \in K_{*}(\mathcal{B}), y \in K_{*}(\mathcal{A})$, the map (2.3) identifies with the transfer map $K_{*}^{t r}(F)$ (1.6), and the projection formula (2.6) holds in $K_{*}(\mathcal{A})$ and similarly for $E=\mathcal{U}_{\mathrm{dg}}^{\text {loc }}$, with $K$ replaced by $\mathbb{K}$. When $F=f$ is as in Example 2.9, we recover Quillen's original projection formula $f_{*}\left(x \cdot f^{*}(y)\right)=f_{*}(x) \cdot y$ on algebraic $K$-theory; see [15, page 103] [25, $\S \mathrm{V} 3.5 .3]$. In the non-connective algebraic $K$-theory case we obtain an analogous projection formula.

Example 2.11 (Hochschild homology). Recall from example [5, Ex. 7.9] the construction of the symmetric monoidal Hochschild homology functor $H H$ : dgcat $\rightarrow$ $\mathcal{D}(k)$. Note that since the base ring $k$ is commutative the category $\mathcal{D}(k)$ carries a natural symmetric monoidal structure with unit object the complex $k$ (concentrated in degree zero). For every small $\mathrm{dg}$ category $\mathcal{C}$ we have a natural isomorphism of $\mathbb{Z}$-graded $k$-modules $\operatorname{Hom}_{*}(k, H H(\mathcal{C})) \simeq H H_{*}(\mathcal{C})$. If $\mathcal{C}$ is moreover endowed with a "multiplication" dg functor as in (2.1), then $H H_{*}(\mathcal{C})$ inherits a multiplicative operation. For instance, if $A$ is a commutative algebra as in Example2.9, the multiplicative operation on $H_{*}(A)$ is given by the shuffle product; see [12, Cor. 4.2.7]. It follows then from Proposition 2.4 applied to $E=H H$ that $x \in H H_{*}(\mathcal{B}), y \in H H_{*}(\mathcal{A})$, 
the map (2.3) identifies with the transfer map $H H_{*}^{t r}(F)$ (1.7), and the projection formula (2.6) holds in $H H_{*}(\mathcal{A})$.

Example 2.12 (Negative cyclic homology). Recall from [5, Ex. 7.10] the construction of the symmetric monoidal mixed complex functor $C:$ dgcat $\rightarrow \mathcal{D}(\Lambda)$. Here, $\Lambda$ is the $k$-algebra $k[\epsilon] / \epsilon^{2}$ with $\epsilon$ of degree -1 and $d(\epsilon)=0$. The derived category $\mathcal{D}(\Lambda)$ carries a symmetric monoidal structure defined on the underlying complexes. The unit object is the complex $k$ endowed with the trivial action of $\epsilon$. For every small dg category $\mathcal{C}$ we have a natural isomorphism of $\mathbb{Z}$-graded $k$-modules $\operatorname{Hom}_{*}(k, C(\mathcal{C})) \simeq H N_{*}(\mathcal{C})$. If $\mathcal{C}$ is moreover endowed with a "multiplication" $\mathrm{dg}$ functor as in (2.1), then $H N_{*}(\mathcal{C})$ inherits a multiplicative operation. For instance, if $A$ is a commutative algebra as in Example 2.9, the multiplicative operation on $H N_{*}(A)$ is given in terms of the shuffle product; see [12, §5.1.13]. It follows then from Proposition 2.4 applied to $E=C$ that $x \in H N_{*}(\mathcal{B}), y \in H N_{*}(\mathcal{A})$, the map (2.3) identifies with the transfer map $H N_{*}^{t r}(F)$ (1.8), and the projection formula (2.6) holds in $H N_{*}(\mathcal{A})$.

Example 2.13 (Periodic cyclic homology). Recall from [5, Ex. 7.11] the construction of the symmetric monoidal periodic complex functor $P: \mathcal{D}(\Lambda) \rightarrow \mathcal{D}(k[u]$ Comod). Here, $k[u]$-Comod is the category of $k[u]$-comodules, where $k[u]$ is the cocommutative Hopf algebra of polynomials in one variable $u$ of degree 2 . The derived category $\mathcal{D}(k[u]$-Comod $)$ carries a symmetric monoidal structure given by the cotensor product of comodules, with unit object $k[u]$. For every small dg category $\mathcal{C}$ we have a natural isomorphism of $\mathbb{Z}$-graded $k$-modules $\operatorname{Hom}_{*}(k,(P \circ C)(\mathcal{C})) \simeq H P_{*}(\mathcal{C})$. If moreover $\mathcal{C}$ is endowed with a "multiplication" $\operatorname{dg}$ functor as in (2.1), then $H P_{*}(\mathcal{C})$ inherits a multiplicative operation. For instance, if $A$ is a commutative algebra as in Example 2.9. then the multiplicative operation on $H P_{*}(A)$ is the one described in [12, §5.1.13] in terms of shuffle products. It follows then from Proposition 2.4 applied to $E=P \circ C$ that $x \in H P_{*}(\mathcal{B}), y \in H P_{*}(\mathcal{A})$, the map (2.3) identifies with the transfer map $H P_{*}^{t r}(F)(1.8)$, and the projection formula (2.6) holds in $H P_{*}(\mathcal{A})$.

\section{SCHEMES}

In this section we assume that our base commutative ring $k$ is a field. By a scheme we mean a quasi-compact and quasi-separated $k$-scheme; see [7].

Given a scheme $X$, we have a Grothendieck category $\mathrm{QCoh}(X)$ of quasi-coherent sheaves of $\mathcal{O}_{X}$-modules and an associated dg category $\mathcal{C}_{\mathrm{dg}}(\mathrm{QCoh}(X))$ of unbounded cochain complexes on $\mathrm{QCoh}(X)$. Let $\mathcal{C}_{\text {parf }}^{\mathrm{dg}}(X)$ be the full small $\mathrm{dg}$ subcategory of $\mathcal{C}_{\mathrm{dg}}(\mathrm{QCoh}(X))$ consisting of those perfect complexes of $\mathcal{O}_{X}$-modules (see [23, §2]) which are bounded above, degreewise flat, and whose stalks have cardinality at most the cardinality of $k$. We will denote by $\mathcal{D}_{\text {parf }}^{\text {dg }}(X)$ the Drinfeld DG quotient [6] (see also [20]) of $\mathcal{C}_{\text {parf }}^{\mathrm{dg}}(X)$ by its full dg subcategory of acyclic complexes; note that since we are working over a field, the homotopical flatness conditions [ 6 , $\S 3.3]$ are automatically fulfilled. The small dg category $\mathcal{D}_{\text {parf }}^{\mathrm{dg}}(X)$ enhances the derived category $\mathcal{D}_{\text {parf }}(X)$ of perfect complexes of $\mathcal{O}_{X}$-modules in the sense that we have a natural equivalence of triangulated categories $\mathrm{H}^{0}\left(\mathcal{D}_{\text {parf }}^{\mathrm{dg}}(X)\right) \simeq \mathcal{D}_{\text {parf }}(X)$; see [13] for the uniqueness of this dg-enhancement. Given a morphism of schemes $f: X \rightarrow Y$ we have a dg-enhancement $f^{*}: \mathcal{D}_{\text {parf }}^{\mathrm{dg}}(Y) \rightarrow \mathcal{D}_{\text {parf }}^{\mathrm{dg}}(X)$ of the derived inverse image functor $\mathbb{L} f^{*}: \mathcal{D}_{\text {parf }}(Y) \rightarrow \mathcal{D}_{\text {parf }}(X)$. 
Lemma 3.1. Let $f: X \rightarrow Y$ be a perfect projective morphism, a flat proper morphism, or a proper morphism of finite Tor-dimension between Noetherian schemes; see [7], 23, §3]. Then, the dg functor $f^{*}: \mathcal{D}_{\text {parf }}^{\mathrm{dg}}(Y) \rightarrow \mathcal{D}_{\text {parf }}^{\mathrm{dg}}(X)$ belongs to $\mathcal{T} r$.

Proof. Recall from the proof of Theorem 1.3 that the dg functor

$$
f^{*}: \mathcal{D}_{\text {parf }}^{\mathrm{dg}}(Y) \longrightarrow \mathcal{D}_{\text {parf }}^{\mathrm{dg}}(X)
$$

belongs to $\mathcal{T} r$ if and only if the bimodule $f^{*} X$ belongs to $\operatorname{rep}\left(\mathcal{D}_{\text {parf }}^{\mathrm{dg}}(X)\right.$, $\left.\mathcal{D}_{\text {parf }}^{\text {dg }}(Y)\right)$. By tensoring with the bimodule $f^{*} X$ we obtain a dg functor (see [9, $\S 3.8])$

$$
-\otimes_{f^{*}} X: \mathcal{C}_{\text {dg }}\left(\mathcal{D}_{\text {parf }}^{\text {dg }}(X)\right) \longrightarrow \mathcal{C}_{\text {dg }}\left(\mathcal{D}_{\text {parf }}^{\text {dg }}(Y)\right) .
$$

If $f$ is as in Lemma 3.1, then the derived direct image functor

$$
\mathbb{R} f_{*}: \mathcal{D}(X) \longrightarrow \mathcal{D}(Y)
$$

preserves perfect complexes (see [23, §3.16]) and so the left derived functor of (3.2) identifies with (3.3). Since for every perfect complex $\mathcal{E}$ of $\mathcal{O}_{X}$-modules we have a natural isomorphism $\mathcal{E} \otimes_{f^{*}} X \simeq \mathbb{R} f_{*}\left(\mathcal{E}^{\cdot}\right)$ in the derived category $\mathcal{D}_{\text {parf }}(Y)$, we conclude that the bimodule $f^{*} X$ belongs to $\operatorname{rep}\left(\mathcal{D}_{\text {parf }}^{\mathrm{dg}}(X), \mathcal{D}_{\text {parf }}^{\mathrm{dg}}(Y)\right)$. This achieves the proof.

Thanks to the work of Thomason-Trobaugh [23, §3], Schlichting [17, §8, Thm. 5], Keller [11, §5.2], and Blumberg-Mandell [1, Thm. 1.3], the algebraic $K$-theory, the cyclic homology (and all its variants), and even the topological cyclic homology of a scheme $X$ can be recovered from its associated dg category $\mathcal{D}_{\text {parf }}^{\mathrm{dg}}(X)$. Therefore, by combining Lemma 3.1 with (1.6)-(1.9), we obtain transfer maps :

$$
\begin{array}{ll}
f_{*}: K_{*}(X) \rightarrow K_{*}(Y), & f_{*}: \mathbb{K}_{*}(X) \rightarrow \mathbb{K}_{*}(Y), \\
f_{*}: H H_{*}(X) \rightarrow H H_{*}(Y), & f_{*}: H C_{*}(X) \rightarrow H C_{*}(Y), \\
f_{*}: H N_{*}(X) \rightarrow H N_{*}(Y), & f_{*}: H P_{*}(X) \rightarrow H P_{*}(Y), \\
f_{*}: T H H_{*}(X) \rightarrow T H H_{*}(Y), & f_{*}: T C_{*}(X) \rightarrow T C_{*}(Y) .
\end{array}
$$

The transfer map $f_{*}: K_{*}(X) \rightarrow K_{*}(Y)$ coincides with the one originally defined by Quillen [15, $\S 7$ 2.7] and Thomason-Trobaugh [23, §§3.16.4-3.16.6]. Moreover, thanks to Theorem 1.3(ii) and (1.10), the above transfer maps are compatible with the classical higher Chern characters and with the Dennis trace map.

Proposition 3.4. Let $f: X \rightarrow Y$ be a morphism of schemes as in Lemma 3.1 . Then, the dg functor $f^{*}: \mathcal{D}_{\text {parf }}^{\mathrm{dg}}(Y) \rightarrow \mathcal{D}_{\text {parf }}^{\mathrm{dg}}(X)$ satisfies the conditions of Proposition 2.4 .

Proof. The derived inverse image functor

$$
\mathbb{L} f^{*}: \mathcal{D}_{\text {parf }}(Y) \longrightarrow \mathcal{D}_{\text {parf }}(X)
$$

is symmetric monoidal with respect to the derived tensor products $-\otimes_{\mathcal{O}_{Y}}^{\mathbb{L}}$ - and $-\otimes_{\mathcal{O}_{X}}^{\mathbb{L}}-$; see $[23, \S 3.15]$. By lifting these derived tensor products to $\mathcal{D}_{\text {parf }}^{\mathrm{dg}}(Y)$ and $\mathcal{D}_{\text {parf }}^{\mathrm{dg}}(X)$ we observe that the left-hand side diagram in (2.5) (with $\mathcal{A}=\mathcal{D}_{\text {parf }}^{\mathrm{dg}}(Y)$, $\mathcal{B}=\mathcal{D}_{\text {parf }}^{\mathrm{dg}}(X)$, and $\left.F=f^{*}\right)$ commutes. Now, let $\mathcal{E}$ be a perfect complex of $\mathcal{O}_{X^{-}}$ modules and $\mathcal{F}$ a perfect complex of $\mathcal{O}_{Y}$-modules. Thanks to [7, §III 3.7] we have a canonical isomorphism

$$
\mathbb{R} f_{*}(\mathcal{E}) \otimes_{\mathcal{O}_{Y}}^{\mathbb{L}} \mathcal{F} \simeq \mathbb{R} f_{*}\left(\mathcal{E} \cdot \otimes_{\mathcal{O}_{X}}^{\mathbb{L}} \mathbb{L} f^{*}\left(\mathcal{F}^{*}\right)\right)
$$


in the derived category $\mathcal{D}_{\text {parf }}(Y)$. The left-hand side of (3.5) identifies with the evaluation of the bimodule $m_{\mathcal{A}} \circ\left[{ }_{F} X\right] \otimes \mathbb{L}$ id at $\mathcal{E} \otimes \mathcal{F}$. The right-hand side of (3.5) identifies with the evaluation of the bimodule $\left[{ }_{F} X\right] \circ m_{\mathcal{B}} \circ$ id $\otimes{ }^{\mathbb{L}}\left[X_{F}\right]$ at $\mathcal{E} \cdot \otimes \mathcal{F}$. Therefore, the right-hand side diagram in (2.5) commutes, and so the proof is finished.

By combining Proposition 3.4 with Examples 2.10 2.13, we obtain the following projection formula:

$$
f_{*}\left(x \cdot f^{*}(y)\right)=f_{*}(x) \cdot y
$$

which holds in $K_{*}(Y), \mathbb{K}_{*}(Y), H H_{*}(Y), H N_{*}(Y)$ and $H P_{*}(Y)$. In the connective algebraic $K$-theory case we then recover the projection formula originally developed by Quillen [15, Prop. 2.10] and Thomason-Trobaugh [23, Prop. 3.17].

\section{Appendix A. BACKGROUND ON DG (BI)MODUleS}

Let $\mathcal{C}(k)$ be the category of (unbounded) cochain complexes of $k$-modules. A differential graded $(=d g)$ category (over the base ring $k$ ) is a $\mathcal{C}(k)$-category and a $d g$ functor is a $\mathcal{C}(k)$-functor; see [3, Defs. 6.2.1-6.2.3].

Let $\mathcal{A}$ be a small dg category. The opposite $\operatorname{dg}$ category $\mathcal{A}^{\text {op }}$ of $\mathcal{A}$ has the same objects as $\mathcal{A}$ and complexes of morphisms given by $\mathcal{A}^{\mathrm{op}}(x, y):=\mathcal{A}(y, x)$. A right $\mathcal{A}$-module (or simply an $\mathcal{A}$-module) is a dg functor $\mathcal{A}^{\mathrm{op}} \rightarrow \mathcal{C}_{\mathrm{dg}}(k)$ with values in the dg category $\mathcal{C}_{\mathrm{dg}}(k)$ of complexes of $k$-modules. We denote by $\mathcal{C}(\mathcal{A})$ the category of $\mathcal{A}$-modules; see [9, §2.3]. The derived category $\mathcal{D}(\mathcal{A})$ of $\mathcal{A}$ is the localization of $\mathcal{C}(\mathcal{A})$ with respect to the class of objectwise quasi-isomorphisms. As explained in [9, §3.1] the differential graded structure of $\mathcal{C}_{\mathrm{dg}}(k)$ makes $\mathcal{C}(\mathcal{A})$ naturally into a $\mathrm{dg}$ category $\mathcal{C}_{\mathrm{dg}}(\mathcal{A})$.

Now, let $\mathcal{A}$ and $\mathcal{B}$ be two small $\mathrm{dg}$ categories. Their tensor product $\mathcal{A} \otimes \mathcal{B}$ is defined as follows: the set of objects is the Cartesian product of the sets of objects of $\mathcal{A}$ and $\mathcal{B}$ and for every two objects $(x, z)$ and $(y, w)$ in $\mathcal{A} \otimes \mathcal{B}$ we have $\mathcal{A} \otimes \mathcal{B}((x, z),(y, w)):=\mathcal{A}(x, y) \otimes \mathcal{B}(z, w)$. The tensor product of dg categories gives rise to a symmetric monoidal structure on the category dgcat of small $\mathrm{dg}$ categories. The unit object is the dg category $\underline{k}$ with one object and with $k$ as the dg algebra of endomorphisms. Finally, by an $\mathcal{A}$ - $\mathcal{B}$-bimodule we mean a dg functor $\mathcal{A} \otimes \mathcal{B}^{\mathrm{op}} \rightarrow \mathcal{C}_{\mathrm{dg}}(k)$.

\section{ACKNOWLEDGMENTS}

The author is very grateful to Andrei Suslin for bringing to his attention the importance of developing a unified treatment of transfer maps and projection formulas. He would also like to thank the anonymous referee for comments and suggestions which greatly improved this paper. Finally, he would like to thank the Department of Mathematics at UIC (where this work was carried out) for its hospitality and the Midwest Topology Network for financial support.

\section{REFERENCES}

[1] A. Blumberg and M. Mandell, Localization theorems in topological Hochschild homology and topological cyclic homology. Available at arXiv:0802.3938.

[2] M. Bökstedt, W. Hsiang, and I. Madsen, The cyclotomic trace and algebraic K-theory of spaces. Invent. Math. 111 (1993), no. 3, 465-539. MR1202133 (94g:55011)

[3] F. Borceux, Handbook of categorical algebra. 2. Encyclopedia of Mathematics and its Applications 51 (1994). Cambridge Univ. Press. MR1313497 (96g:18001b) 
[4] D.-C. Cisinski and G. Tabuada, Non-connective K-theory via universal invariants. Compositio Mathematica 147 (2011), 1281-1320.

[5] _ Symmetric monoidal structure on non-commutative motives. Available at arXiv:1001.0228v2. To appear in J. of $K$-Theory.

[6] V. Drinfeld, DG quotients of DG categories. J. Algebra 272 (2004), 643-691. MR 2028075 (2006e:18018)

[7] A. Grothendieck, Théorie des intersections et théorème de Riemann-Roch. Séminaire de Géométrie Algébrique du Bois-Marie 1966-1967 (SGA 6). Lecture Notes in Mathematics 225 (1971).

[8] L. Hesselholt and I. Madsen, On the K-theory of local fields. Ann. of Math. (2) 158 (2003), no. 1, 1-113. MR.1998478(2004k:19003)

[9] B. Keller, On differential graded categories. International Congress of Mathematicians (Madrid), Vol. II (2006), 151-190. Eur. Math. Soc., Zürich. MR2275593 (2008g:18015)

[10] _ On the cyclic homology of exact categories. J. Pure Appl. Alg. 136(1) (1999), 1-56. MR:1667558 (99m:18012)

[11] - On the cyclic homology of ringed spaces and schemes. Doc. Math. 3 (1998), 231-259. MR.1647519 (99i:16018)

[12] J.-L. Loday, Cyclic homology. Grundlehren der Mathematischen Wissenschaften 301 (1992). Springer-Verlag, Berlin. MR1217970 (94a:19004)

[13] V. Lunts and D. Orlov, Uniqueness of enhancement for triangulated categories. J. Amer. Math. Soc. 23 (2010), 853-908. MR2629991

[14] A. Neeman, Triangulated categories. Ann. Math. Studies 148 (2001). Princeton Univ. Press. MR 1812507 (2001k:18010)

[15] D. Quillen, Higher algebraic K-theory. I. Lecture Notes in Mathematics 341 (1973), 85-147. Springer, Berlin. MR0338129(49:2895)

[16] C. Schlichtkrull, Transfer maps and the cyclotomic trace. Math. Ann. 336 (2006), no. 1, 191-238. MR2242623(2007c:16019)

[17] M. Schlichting, Negative K-theory of derived categories. Math. Z. 253 (2006), no. 1, 97-134. MR2206639 (2006i:19003)

[18] G. Tabuada, Higher K-theory via universal invariants. Duke Math. J. 145 (2008), no. 1, 121-206. MR2451292 (2009j:18014)

[19] _ Invariants additifs de dg-catégories. Int. Math. Res. Not. 53 (2005), 3309-3339. MR2196100 (2006k:18018)

[20] , On Drinfeld's DG quotient. J. Algebra 323 (2010), 1226-1240. MR 2584954 (2011a:16022)

[21] - Generalized spectral categories, topological Hochschild homology, and trace maps. Algebraic and Geometric Topology 10 (2010), 137-213. MR 2580431(2011a:55012)

$[22]$ _ Products, multiplicative Chern characters, and finite coefficients via noncommutative motives. Available at arXiv:1101.0731.

[23] R. W. Thomason and T. Trobaugh, Higher algebraic K-theory of schemes and of derived categories. Grothendieck Festschrift, Volume III. Volume 88 of Progress in Math., 247-436. Birkhäuser, Boston 1990. MR 1106918 (92f:19001)

[24] B. Toën, The homotopy theory of dg-categories and derived Morita theory. Invent. Math. 167 (2007), no. 3, 615-667. MR.2276263 (2008a:18006)

[25] C. Weibel, The K-book: An introduction to algebraic $K$-theory. A graduate textbook in progress. Available at http://www.math.rutgers.edu/weibel/Kbook.html.

Departamento de Matemática e CMA, FCT-UnL, Quinta da Torre, 2829-516 Caparica, Portugal

E-mail address: tabuada@fct.unl.pt

Current address: Department of Mathematics, Massachusetts Institute of Technology, Cambridge, Massachusetts 02139

E-mail address: tabuada@math.mit.edu 